\title{
GEODESIC UNIQUENESS AND DERIVATIVES OF BERS PROJECTION
}

\begin{abstract}
Hui Guo
In this paper, we discover a sufficient and necessary condition under which two geodesic segments joining the basepoint and another point in an infinitedimensional Teichmüller space are the same.
\end{abstract}

\section{INTRODUCTION}

A metric space is said to be a straight geodesic space in the sense of Busemann ([1]) if for any two (distinct) points in it there is a unique geodesic segment joining them and such a segment can be uniquely extended to a straight line. Here by "straight line" we mean the image of an isometric imbedding of the real line $\mathbb{R}$ into the given metric space with respect to the Euclidean metric and the metric of the given metric space respectively. Similarly, by "geodesic segment" we mean the image of a closed interval of $\mathbb{R}$.

It is well known that a finite-dimensional Teichmüller space is a straight geodesic space in the sense of Busemann ([5]). A very natural and basic problem is whether or not an infinite-dimensional Teichmüller space is a straight geodesic space in the sense of Busemann.

Let $[\mu](\neq[0])$ be a point in a Teichmüller space $T(\Gamma)$. When $T(\Gamma)$ is finitedimensional, the extremal Beltrami differential in $[\mu]$ is unique, say $\mu_{0}$; then $\mu_{0}$ must be a Teichmüller differential and the path $\left[t \mu_{0}\right](0 \leqslant t \leqslant 1)$ is the unique geodesic segment joining [0] and $[\mu]$. However, when $T(\Gamma)$ is infinite-dimensional, $[\mu]$ can contain more than one extremal Beltrami differentials. The first example of such a point in the universal Teichmüller space was given by Strebel, known as the Strebel's chimney ([12]).

In order to determine whether or not an infinite-dimensional Teichmüller space is a straight geodesic space in the sense of Busemann, one can investigate the following basic question proposed by Gardiner ([3]).

Received 16th July, 2002

Supported partly by the National Natural Science Foundation of China (Grant No. 19801024) and the Natural Science Foundation of Guangdong Province in China (Grant No. 984112).

The author would like to give his thanks to the Institute of Mathematics, Academy of Mathematics and System Sciences, Chinese Academy of Sciences, P. R. China and the University of Central Lancashire, UK for their hospitality during his visiting time.

Copyright Clearance Centre, Inc. Serial-fee code: 0004-9727/03 \$A2.00+0.00. 
Gardiner's Problem. Suppose $T(\Gamma)$ is an infinite-dimensional Teichmüller space and $[\mu] \in T(\Gamma)$ contains two extremal Beltrami differentials $\mu_{1}$ and $\mu_{2}$. Are the paths

$$
\alpha_{1}:[0,1] \rightarrow T(\Gamma), t \mapsto\left[t \mu_{1}\right]
$$

and

$$
\alpha_{2}:[0,1] \rightarrow T(\Gamma), t \mapsto\left[t \mu_{2}\right]
$$

the same?

It is well known that the images of both $\alpha_{1}$ and $\alpha_{2}$ are geodesic segments joining [0] and $[\mu]$ because of extremality of $\mu_{1}$ and $\mu_{2}$ ([10]). With respect to Gardiner's problem, $\mathrm{Li}([8])$ has first given an example for $\alpha_{1} \neq \alpha_{2}$ in the universal Teichmüller space, where $\alpha_{1} \neq \alpha_{2}$ means the two paths (or geodesic segments) $\alpha_{1}$ and $\alpha_{2}$ are not the same. Later, Tanigawa $([\mathbf{1 3}])$ and $\mathrm{Li}([\mathbf{9}])$ respectively gave some examples for $\alpha_{1} \neq \alpha_{2}$ in an infinite-dimensional Teichmüller space, which gave a negative answer to Gardiner's problem. These results lead to the fact that any infinite-dimensional Teichmüller space is not a straight geodesic space in the sense of Busemann. Further, one would like to ask when $\alpha_{1} \neq \alpha_{2}$ and when $\alpha_{1}=\alpha_{2}$. In fact, Tanigawa ([13]), Li ([9]) and Shen ([11]) have given some sufficient conditions for $\alpha_{1} \neq \alpha_{2}$ in an infinitedimensional Teichmüller space. The "best (or weakest)" one is the following:

THEOREM A. ([9]) Let $T(\Gamma)$ be an infinite-dimensional Teichmüller space and $\mu_{1}, \mu_{2}$ be two extremal Beltrami differentials in a point $[\mu]$ of $T(\Gamma)$. If $\mu_{1}-\mu_{2} \notin N(\Gamma)$, then $\left[t \mu_{1}\right](0 \leqslant t \leqslant 1)$ and $\left[t \mu_{2}\right](0 \leqslant t \leqslant 1)$ are two different geodesic segments joining $[0]$ and $[\mu]$ in $T(\Gamma)$, where $N(\Gamma)$ is the Ahlfors $N$-class of $\Gamma$.

In this paper, we discover a sufficient and necessary condition for $\alpha_{1} \neq \alpha_{2}$ in an infinite-dimensional Teichmüller space. That is the following main theorem:

Theorem 1. Let $T(\Gamma)$ be an infinite-dimensional Teichmüller space and $\mu_{1}, \mu_{2}$ be two extremal Beltrami differentials in a point $[\mu]$ of $T(\Gamma)$. Then $\left[t \mu_{1}\right](0 \leqslant t \leqslant 1)$ and $\left[t \mu_{2}\right](0 \leqslant t \leqslant 1)$ are the same geodesic segments joining $[0]$ and $[\mu]$ in $T(\Gamma)$ if and only if

$$
\mu_{1}-\mu_{2} \in N(\Gamma)
$$

and

$$
\mu_{1} \underbrace{H\left(\mu _ { 1 } H \left(\cdots \left(\mu_{1} H\right.\right.\right.}_{k}\left(\mu_{1}\right))))-\mu_{2} \underbrace{H\left(\mu _ { 2 } H \left(\cdots \left(\mu_{2} H\right.\right.\right.}_{k}\left(\mu_{2}\right)))) \in N^{*}(1), k=1,2, \ldots,
$$

where $H$ is the Hilbert transformation, $N(\Gamma)$ is the Ahlfors $N$-class of $\Gamma$ and $N^{*}(1)$ is the Ahlfors quasi- $N$-class of $\left\{i_{d}\right\}$.

Corollary. Let $T(1)$ be the universal Teichmüller space and $\mu_{1}, \mu_{2}$ be two extremal Beltrami differentials in a point $[\mu]$ of $T(1)$. Then $\left[t \mu_{1}\right](0 \leqslant t \leqslant 1)$ and 
$\left[t \mu_{2}\right](0 \leqslant t \leqslant 1)$ are the same geodesic segments joining $[0]$ and $[\mu]$ in $T(1)$ if and only if

$$
\mu_{1}-\mu_{2} \in N(1)
$$

and

$$
\mu_{1} \underbrace{H\left(\mu _ { 1 } H \left(\cdots \left(\mu_{1} H\right.\right.\right.}_{k}\left(\mu_{1}\right))))-\mu_{2} \underbrace{H\left(\mu _ { 2 } H \left(\cdots \left(\mu_{2} H\right.\right.\right.}_{k}\left(\mu_{2}\right)))) \in N^{*}(1), k=1,2, \ldots,
$$

where $N(1)$ is the Ahlfors $N$-class of $\left\{i_{d}\right\}$.

\section{Preliminaries}

Let $w(z) \in L_{0}^{p}(\mathbb{C})$, where $p>2$ and

$$
L_{0}^{p}(\mathbb{C})=\left\{w(z) \in L^{p}(\mathbb{C}): w(z) \text { has compact support }\right\} .
$$

We define a linear operator $T$ on $L_{0}^{p}(\mathbb{C})$ :

$$
T w(z)=-\frac{1}{\pi} \iint_{\mathrm{C}} \frac{w(\zeta)}{\zeta-z} d \xi d \eta, \quad \zeta=\xi+i \eta
$$

It is evident that $T w(z)$ is holomorphic in $\mathbb{C} \backslash D$ and

$$
T w(z)=O\left(\frac{1}{|z|}\right), \text { when } z \rightarrow \infty,
$$

where $D$ is the support of $w(z)$ (see [7]).

Let $w(z) \in C_{0}^{\infty}(\mathbb{C})$. We introduce another linear operator $H$ on $C_{0}^{\infty}(\mathbb{C})$, which is called the Hilbert transformation:

$$
H w(z)=-\frac{1}{\pi} \iint_{\mathbf{C}} \frac{w(\zeta)-w(z)}{(\zeta-z)^{2}} d \xi d \eta, \quad \zeta=\xi+i \eta
$$

The Hilbert transformation can be extended as a bounded linear operator of $L^{p}(\mathbb{C})$ into itself, where $1<p<+\infty$ (see [6] or [7]). We denote by $\Lambda_{p}$ the norm of the operator $H$, that is,

$$
\Lambda_{p}=\sup _{w \in L^{p}(\mathbf{C})}\left\{\|H w(z)\|_{p} /\|w(z)\|_{p}\right\}, \quad 1<p<+\infty .
$$

Then $\Lambda_{p}$ is continuous with respect to $p$ and $\Lambda_{2}=1$ (see [6] or [7]).

Let $S$ be a Riemann surface which has a universal covering $\Delta$, where $\Delta$ is the open unit disc. Then the Riemann surface $S$ can be represented as $\Delta / \Gamma$, where $\Gamma$ is a torsion free Fuchsian group acting on $\Delta$. If $S=\Delta$, then $\Gamma$ is trivial, that is, $\Gamma=\left\{i_{d}\right\}$. 
We denote by Belt $(\Gamma)$ the Banach space of all the Beltrami differentials of $\Gamma$ with finite $L^{\infty}$-norm:

$\operatorname{Belt}(\Gamma)=\left\{\mu \in L^{\infty}(\Delta): \forall \gamma \in \Gamma, \mu(\gamma(z)) \overline{\gamma^{\prime}(z)} / \gamma^{\prime}(z)=\mu(z)\right.$ almost everywhere in $\left.\Delta\right\}$

Denote by $B(\Gamma)$ the open unit ball of Belt $(\Gamma)$ and by $f^{\mu}: \Delta \rightarrow \Delta$ the quasiconformal mapping with the Beltrami coefficient $\mu$ and keeping $-1, i$ and 1 fixed. We introduce a kind of equivalence relationship in $B(\Gamma)$. We say that $\mu_{1}$ is equivalent to $\mu_{2}$ if and only if

$$
\left.\left.f^{\mu_{1}}\right|_{\partial \Delta} \equiv f^{\mu_{2}}\right|_{\partial \Delta}
$$

where $\partial \Delta$ is the boundary of $\Delta$. Then the Teichmüller space of $S$ (or $\Gamma$ ), denoted by $T(S)$ (or $T(\Gamma)$ ), is defined as the set of all the equivalence class of the elements in $B(\Gamma)$. When $\Gamma$ is of the second kind or infinitely generated, $T(\Gamma)$ is infinite-dimensional. When $\Gamma$ is trivial, the corresponding Teichmüller space, denoted by $T(1)$, is called the universal Teichmüller space.

A Beltrami differential $\mu \in B(\Gamma)$ is said to be extremal if and only if

$$
\|\mu\|_{\infty} \leqslant\|\tilde{\mu}\|_{\infty}, \quad \forall \tilde{\mu} \in[\mu]
$$

where $[\mu]$ is the equivalence class of $\mu$. It is well known that $\mu \in B(\Gamma)$ is extremal if and only if it satisfies the Hamilton-Krushkal condition:

$$
\sup _{\phi \in S Q D(\Gamma)} \iint_{\Delta / \Gamma} \mu \phi d x d y=\|\mu\|_{\infty}
$$

where $S Q D(\Gamma)$ is the unit sphere of the Banach space of all the holomorphic quadratic differentials of $\Gamma$ with finite $L^{1}$-norm.

The Teichmüller metric for $T(\Gamma)$ is defined in terms of the extremal Beltrami differentials. For any given two points $\left[\mu_{1}\right]$ and $\left[\mu_{2}\right]$ in $T(\Gamma)$, the Teichmüller distance between them is

$$
d_{T}\left(\left[\mu_{1}\right],\left[\mu_{2}\right]\right)=\frac{1}{2} \log \frac{1+\|\mu\|_{\infty}}{1-\|\mu\|_{\infty}}
$$

where $\mu$ is an extremal Beltrami differential in the equivalence class of the Beltrami coefficient of $f^{\mu_{1}} \circ\left(f^{\mu_{2}}\right)^{-1}$. It is well known that the Teichmüler metric is complete and coincides with the Kobayashi metric.

The Ahlfors $N$-class of $\Gamma$ is defined as follows:

$$
N(\Gamma)=\left\{\mu \in \operatorname{Belt}(\Gamma): \iint_{\Delta / \Gamma} \mu \phi d x d y=0, \forall \phi \in Q D(\Gamma)\right\}
$$


where $Q D(\Gamma)$ is the Banach space of all the holomorphic quadratic differentials of $\Gamma$ with finite $L^{1}$-norm, that is,

$$
\begin{aligned}
& Q D(\Gamma)=\left\{\phi \in L^{1}(\Delta / \Gamma): \phi \text { is holomorphic in } \Delta ;\right. \\
& \left.\qquad \gamma \in \Gamma, \phi(\gamma(z))\left(\gamma^{\prime}(z)\right)^{2}=\phi(z) \text { in } \Delta\right\} .
\end{aligned}
$$

Especially, when $\Gamma$ is trivial, that is, $\Gamma=\left\{i_{d}\right\}$,

$$
N(1)=\left\{\mu \in \operatorname{Belt}(1): \iint_{\Delta} \mu \phi d x d y=0, \forall \phi \in Q D(1)\right\},
$$

where

$$
Q D(1)=\left\{\phi \in L^{1}(\Delta): \phi \text { is holomorphic in } \Delta\right\} .
$$

Let $1<p<+\infty$. The Ahlfors $N^{p}$-class of $\left\{i_{d}\right\}$ is defined as follows:

$$
N^{p}(1)=\left\{\mu \in L^{p}(\Delta): \iint_{\Delta} \mu \phi d x d y=0, \forall \phi \in Q D^{q}(1)\right\}
$$

where

$$
\begin{aligned}
Q D^{q}(1) & =Q D(1) \bigcap L^{q}(\Delta), \\
q & =\frac{p}{p-1}
\end{aligned}
$$

The Ahlfors quasi- $N$-class of $\left\{i_{d}\right\}$ is defined as follows:

$$
N^{*}(1)=\left\{\mu \in \operatorname{Belt}^{*}(1): \iint_{\Delta} \mu \phi d x d y=0, \forall \phi \in Q D^{*}(1)\right\}
$$

where

$$
\begin{aligned}
\operatorname{Belt}^{*}(1) & =\bigcap_{1<p<+\infty} L^{p}(\Delta), \\
Q D^{*}(1) & =\bigcup_{1<q<+\infty} Q D^{q}(1) .
\end{aligned}
$$

It is evident that $N(1) \subseteq N^{*}(1)$.

Let $X$ and $Y$ be two Banach spaces and $F$ an operator from $U$ to $Y$, where $U$ is an open set of $X . F$ is said to be Gâteaux differentiable at $x_{0} \in U$ if there is an operator $d F\left(x_{0} ; \cdot\right)$ from $X$ to $Y$ such that

$$
\left\|F\left(x_{0}+t h\right)-F\left(x_{0}\right)-t \cdot d F\left(x_{0} ; h\right)\right\|=o(t)
$$


when $|t| \rightarrow 0+$ and $x_{0}+t h \in U$. In this case, $d F\left(x_{0} ; h\right)$ is called the Gâteaux derivative of $F$ at $x_{0}$ along the direction $h$ and clearly

$$
d F\left(x_{0} ; h\right)=\lim _{|t| \rightarrow 0+} \frac{F\left(x_{0}+t h\right)-F\left(x_{0}\right)}{t} .
$$

$F$ is said to be Gâteaux differentiable on $U$ if $F$ is Gâteaux differentiable at each point $x \in U$.

The Gâteaux differentiability and Gâteaux derivatives $d^{n} F\left(x_{0} ; h_{1}, \ldots, h_{n}\right)$ of higher order $(n \geqslant 2)$ can be defined successively. For example, a Gâteaux differentiable operator $F$ from $U$ to $Y$ is said to be twice Gâteaux differentiable at $x_{0}$ if $d F\left(x ; h_{1}\right)$ from $U$ to $Y$ is Gâteaux differentiable at $x_{0}$ for any fixed $h_{1} \in X$. In this case, $d^{2} F\left(x_{0} ; h_{1}, h_{2}\right)$ is called the twice Gâteaux derivative at $x_{0}$ along the directions $h_{1}$ and $h_{2}$. Clearly,

$$
d^{2} F\left(x_{0} ; h_{1}, h_{2}\right)=\lim _{|t| \rightarrow 0+} \frac{d F\left(x_{0}+t h_{2} ; h_{1}\right)-d F\left(x_{0} ; h_{1}\right)}{t} .
$$

$F$ is said to be twice Gâteaux differentiable on $U$ if $F$ is twice Gâteaux differentiable at each point $x \in U$.

\section{LeMmaS}

Suppose that $\mu \in L_{0}^{\infty}(\mathbb{C})$, where

$$
L_{0}^{\infty}(\mathbb{C})=\left\{\mu(z) \in L^{\infty}(\mathbb{C}): \mu(z) \text { has compact support }\right\}
$$

We defined inductively

$$
\varphi_{1}=\mu, \quad \varphi_{n}=\mu H\left(\varphi_{n-1}\right), \quad n=2,3, \ldots,
$$

where $H$ is the Hilbert transformation.

LEMMA 1. (Representation Theorem) Let $f$ be a quasiconformal mapping of the plane with Beltrami coefficient $\mu \in L_{0}^{\infty}(\mathbb{C})$ and satisfying the normalisation condition: $\lim _{z \rightarrow \infty}(f(z)-z)=0$. Then

$$
f(z)=z+\sum_{i=1}^{+\infty} T \varphi_{i}(z), \quad \forall z \in \mathbb{C},
$$

where $\varphi_{i}$ is defined by (1). The series is absolutely and uniformly convergent in the plane.

ProOF: See [6] or [7]. 
Lemma 2. (Uniqueness Theorem) Let $f$ and $g$ be two quasiconformal mappings of a domain $D$ into $\mathbb{C}$ whose Beltrami coefficients agree almost everywhere in $D$. Then $f \circ g^{-1}$ is a conformal mapping.

Proof: See [6] or [7].

LEMmA 3. Let $\mu \in L_{0}^{\infty}(\mathbb{C})$ and $\|\mu\|_{\infty}<1$. Let $z \mapsto f(z, t)$ be the quasiconformal mapping of the plane with Beltrami coefficient $t \mu\left(|t|<1 /\|\mu\|_{\infty}\right)$ and with the property: $\lim _{z \rightarrow \infty}(f(z, t)-z)=0$. Then, for any fixed $z \neq \infty$, the function $t \mapsto f(z, t)$ is holomorphic in the disc $\left\{t \in \mathbb{C}:|t|<1 /\|\mu\|_{\infty}\right\}$.

Also, for any fixed $z$ outside the support of $\mu$, the derivatives of the analytic function $z \mapsto f(z, t)$ depend holomorphically on $t$ in the disc $\left\{t \in \mathbb{C}:|t|<1 /\|\mu\|_{\infty}\right\}$.

Proof: See [6] or [7].

Let $f$ be meromorphic and locally injective in a domain $D$. We define the Schwarzian derivative of the function $f$ as follows:

$$
S_{f}=\left(\frac{f^{\prime \prime}}{f^{\prime}}\right)^{\prime}-\frac{1}{2}\left(\frac{f^{\prime \prime}}{f^{\prime}}\right)^{2}
$$

It is easy to see that $S_{f}$ is holomorphic in $D$.

Let $\mu \in B(\Gamma)$ and $\Delta^{*}=\{z \in \mathbb{C}:|z|>1\}$. We denote by $f_{\mu}$ the quasiconformal mapping of the plane whose Beltrami coefficient equals $\mu$ in $\Delta$ and zero in $\Delta^{*}$ and which keeps $-1, i$ and 1 fixed. Then $\left.f_{\mu}\right|_{\Delta^{*}}$ is a univalent function. Set $\Phi(\mu)=S_{\left.f_{\mu}\right|_{\Delta^{*}}}$, where $S_{\left.f_{\mu}\right|_{\Delta^{*}}}$ is the Schwarzian derivative of $\left.f_{\mu}\right|_{\Delta^{*}}$. This describes a mapping $\Phi$ of $B(\Gamma)$ into $H Q D\left(\Gamma^{*}\right): \mu \mapsto \Phi(\mu)$, called Bers projection, where $H Q D\left(\Gamma^{*}\right)$ is the Banach space of all the holomorphic quadratic differentials of $\Gamma^{*}$ with finite hyperbolic sup-norm:

$$
\|\phi\|_{h}=\sup _{z \in \Delta^{*}}\left(|z|^{2}-1\right)^{2}|\phi(z)| / 4
$$

that is,

$$
\begin{aligned}
& H Q D\left(\Gamma^{*}\right)=\left\{\phi(z): \phi(z) \text { is holomorphic in } \Delta^{*} ; \forall \gamma \in \Gamma^{*},\right. \\
& \left.\qquad \phi(\gamma(z))\left(\gamma^{\prime}(z)\right)^{2}=\phi(z) \text { in } \Delta^{*} ;\|\phi\|_{h}<+\infty\right\} .
\end{aligned}
$$

Here $\Gamma^{*}$ is the torsion free Fuchsian group acting on $\Delta^{*}$ which is induced by $\Gamma$, that is,

$$
\Gamma^{*}=\left\{\left.\gamma\right|_{\Delta^{*}}: \gamma \in \Gamma\right\}
$$

The mapping $\Phi$ induces a mapping $\tilde{\Phi}$ of $T(\Gamma)$ into $H Q D\left(\Gamma^{*}\right):[\mu] \mapsto \Phi(\mu)$, called Bers imbedding. 
LEMma 4. (Bers Imbedding) The mapping $\widetilde{\Phi}$ is a homeomorphism of $T(\Gamma)$ onto a bounded domain of $H Q D\left(\Gamma^{*}\right)$.

Proof: See [6] or [7].

Lemma 5. (Nehari) Each univalent function $f$ on $\Delta^{*}$ satisfies the inequality

$$
\left\|S_{f}\right\|_{h} \leqslant \frac{3}{2}
$$

especially it holds for the univalent function $\left.f_{\mu}\right|_{\Delta^{*}}$ induced by $\mu \in B(\Gamma)$.

ProOF: See [6] or [7].

LEMma 6.

(A) Let $\mu \in \operatorname{Belt}(\Gamma)$ and $a(z)=T \mu(z)$ for $z \in \Delta^{*}$. Then the following properties are equivalent:

(A1) $\mu \in N(\Gamma)$;

(A2) There is an integer $k \geqslant 0$ such that the derivative of order $k$ of $a(z)$

$$
a^{(k)}(z) \equiv 0, \quad \forall z \in \Delta^{*} ;
$$

(A3) For any integer $m \geqslant 0$, the derivative of order $m$ of $a(z)$

$$
a^{(m)}(z) \equiv 0, \quad \forall z \in \Delta^{*}
$$

(B) Let $\mu \in \mathrm{Belt}^{*}(1)$ and $a(z)=T \mu(z)$ for $z \in \Delta^{*}$. Then the following properties are equivalent:

(B1) $\mu \in N^{*}(1)$;

(B2) There is an integer $k \geqslant 0$ such that the derivative of order $k$ of $a(z)$

$$
a^{(k)}(z) \equiv 0, \quad \forall z \in \Delta^{*} ;
$$

(B3) For any integer $m \geqslant 0$, the derivative of order $m$ of $a(z)$

$$
a^{(m)}(z) \equiv 0, \quad \forall z \in \Delta^{*}
$$

Proof: (A). It is evident that property (A3) implies property (A2).

Suppose that property (A2) holds. By definition and a straightforward computation, for any $z \in \Delta^{*}$,

$$
a^{(k)}(z)=-\frac{k !}{\pi} \iint_{\Delta} \frac{\mu(\zeta)}{(\zeta-z)^{k+1}} d \xi d \eta, \quad \zeta=\xi+i \eta
$$


Hence

$$
a^{(k)}(z)=\sum_{n=0}^{+\infty} b_{n} z^{-(n+k+1)}
$$

where

$$
b_{n}=(-1)^{k} \frac{(n+1) \cdots(n+k)}{\pi} \iint_{\Delta} \mu(\zeta) \zeta^{n} d \xi d \eta
$$

By supposition and holomorphy of $a(z)$ in $\Delta^{*}$, we have $b_{n}=0, n=0,1, \ldots$, that is,

$$
\iint_{\Delta} \mu(\zeta) \zeta^{n} d \xi d \eta=0, \quad n=0,1, \ldots
$$

By Runge's approximation theorem, (2) are equivalent to the property

$$
\iint_{\Delta} \mu(\zeta) f(\zeta) d \xi d \eta=0
$$

for all holomorphic and $L^{1}$-integrable functions $f$ in $\Delta$ (see [2]).

On the other hand, we have

$$
\begin{aligned}
\iint_{\Delta} \mu(\zeta) f(\zeta) d \xi d \eta & =\sum_{\gamma \in \Gamma} \iint_{\gamma(D)} \mu(\zeta) f(\zeta) d \xi d \eta \\
& =\iint_{D} \mu(\zeta) \Theta f(\zeta) d \xi d \eta \\
& =\iint_{\Delta / \Gamma} \mu(\zeta) \Theta f(\zeta) d \xi d \eta
\end{aligned}
$$

where $D$ is the fundamental domain of $\Gamma$ and $\Theta f$ is the Poincaré theta series of $f$, that is,

$$
\Theta f=\sum_{\gamma \in \Gamma}(f \circ \gamma)\left(\gamma^{\prime}\right)^{2} .
$$

So for all holomorphic and $L^{1}$-integrable functions $f$ in $\Delta$, we have

$$
\iint_{\Delta / \Gamma} \mu(\zeta) \Theta f(\zeta) d \xi d \eta=0
$$

It is a well-known fact that for every $\phi \in Q D(\Gamma)$, there is a holomorphic and $L^{1}$-integrable function $f$ such that $\Theta f=\phi$ (see [6] or [4]). Hence from (4) we obtain

$$
\iint_{\Delta / \Gamma} \mu \phi d x d y=0, \quad \forall \phi \in Q D(\Gamma)
$$


that is, (A2) implies (A1).

Suppose that property (A1) holds. Similarly, by definition and a straightforward computation, we know that for any integer $m \geqslant 0$ and $z \in \Delta^{*}$,

$$
\begin{aligned}
a^{(m)}(z) & =-\frac{m !}{\pi} \iint_{\Delta} \frac{\mu(\zeta)}{(\zeta-z)^{m+1}} d \xi d \eta, \quad \zeta=\xi+i \eta \\
& =\sum_{n=0}^{+\infty} \widetilde{b}_{n} z^{-(n+m+1)}
\end{aligned}
$$

where

$$
\tilde{b}_{n}=(-1)^{m} \frac{(n+1) \cdots(n+m)}{\pi} \iint_{\Delta} \mu(\zeta) \zeta^{n} d \xi d \eta, \quad n=0,1, \ldots
$$

Let $\Theta_{n}$ be the Poincaré theta series of $f(z)=z^{n}$. Then by a computation similar to (3) we have

$$
\iint_{\Delta} \mu(\zeta) \zeta^{n} d \xi d \eta=\iint_{\Delta / \Gamma} \mu(\zeta) \Theta_{n}(\zeta) d \xi d \eta
$$

It is well known that $\Theta f \in Q D(\Gamma)$ for every holomorphic and $L^{1}$-integrable function $f$ in $\Delta$, especially for $f(z)=z^{n}$. Hence by supposition, we know

$$
\widetilde{b}_{n}=0, \quad n=0,1, \ldots
$$

that is, (A1) implies (A3).

(B). Its proof is completely similar to the proof of (A). But now $\Gamma=\left\{i_{d}\right\}$ and the Runge's approximation theorem is used to all the $L^{q}$-integrable $(1<q<+\infty)$ and holomorphic functions defined in $\Delta$ (see [2]).

\section{Derivatives of Bers projection}

It is well known that Bers projection is Fréchet differentiable and has Gâteaux derivatives of high order. In order to be complete, an interesting expression for the Gâteaux derivatives of order $n$ of Bers projection at the origin is given here.

For any $\mu \in B(\Gamma)$, we denote by $\tilde{f}_{\mu}$ the quasiconformal mapping of the plane whose Beltrami coefficient equals $\mu$ in $\Delta$ and zero in $\Delta^{*}$ and which satisfies the normalisation condition: $\lim _{z \rightarrow \infty}\left(\tilde{f}_{\mu}(z)-z\right)=0$. Then $\tilde{f}_{\mu}$ is conformal in $\Delta^{*}$. We define a mapping of $B(\Gamma)$ into $H Q D\left(\Gamma^{*}\right)$

$$
\Psi: \mu \mapsto S_{\left.\tilde{f}_{\mu}\right|_{\Delta}}
$$

where $S_{\left.\tilde{f}_{\mu}\right|_{\Delta^{*}}}$ is the Schwarzian derivative of $\left.\tilde{f}_{\mu}\right|_{\Delta^{*}}$. 
Lemma 7. For any $\mu \in B(\Gamma), \Psi(\mu)=\Phi(\mu)$ in $H Q D\left(\Gamma^{*}\right)$.

Proof: By definition, $f_{\mu}$ and $\tilde{f}_{\mu}$ have the same Beltrami coefficients in $\mathbb{C}$. Then by Lemma 2, $\tilde{f}_{\mu} \circ\left(f_{\mu}\right)^{-1}$ is a conformal mapping in $\mathbb{C}$, that is, $\tilde{f}_{\mu} \circ\left(f_{\mu}\right)^{-1}$ is a Möbius transformation. Let $h=\tilde{f}_{\mu} \circ\left(f_{\mu}\right)^{-1}$. Then $\left.\tilde{f}_{\mu}\right|_{\Delta^{*}}=\left.h \circ f_{\mu}\right|_{\Delta^{*}}$. Thus

$$
S_{\left.\widetilde{f}_{\mu}\right|_{\Delta^{*}}}=\left(\left.S_{h} \circ f_{\mu}\right|_{\Delta^{*}}\right)\left(\left.f_{\mu}^{\prime}\right|_{\Delta^{*}}\right)^{2}+S_{\left.f_{\mu}\right|_{\Delta^{*}}}
$$

But $S_{h} \equiv 0$ because $h$ is a Möbius transformation. Hence

$$
S_{\left.\widetilde{f}_{\mu}\right|_{\Delta^{*}}}=S_{\left.f_{\mu}\right|_{\Delta^{*}}}
$$

that is, Lemma 7 follows.

Let $\mu \in B(\Gamma)$ be fixed. By Lemma 3, for any fixed $z$ in $\Delta^{*}, \tilde{f}_{t \mu}(z)$ and its derivatives with respect to $z$ all are holomorphic with respect to $t$ in the disc $\Delta_{\mu}$ $=\left\{t \in \mathbb{C}:|t|<1 /\|\mu\|_{\infty}\right\}$. Then for any fixed $z$ in $\Delta^{*}, \Psi(t \mu)(z)$ is holomorphic with respect to $t$ in the disc $\Delta_{\mu}$. Thus by Lemma 7 we obtain that for any fixed $z$ in $\Delta^{*}$, $\Phi(t \mu)(z)$ is holomorphic with respect to $t$ in the disc $\Delta_{\mu}$.

Theorem 2. Let $\mu \in B(\Gamma)$ be fixed. Then for any positive integer $n$ and any $c \in \mathbb{C},|c|<1$, we have

$$
\left\|\Phi(c \mu)-\Phi(0)-d \Phi(0 ; \mu) \cdot c-\cdots-d^{n} \Phi(0 ; \underbrace{\mu, \ldots, \mu}_{n}) \cdot \frac{c^{n}}{n !}\right\|_{h} \leqslant \frac{3}{\delta}|c|^{n+1},
$$

where $d^{k} \Phi(0 ; \underbrace{\mu, \ldots, \mu}_{k}), k=1,2, \ldots, n$, is the Gâteaux derivatives of order $k$ of Bers projection $\Phi$ at the origin along the direction $\mu$ and $\delta$ is a positive constant only depending on $\|\mu\|_{\infty}$.

Proof: By Cauchy's formula, for any fixed $z \in \Delta^{*}$, we have

$$
\Phi(t \mu)(z)=\frac{1}{2 \pi i} \int_{\beta} \frac{\Phi(w \mu)(z)}{w-t} d w, \quad|t|<1+\delta
$$

where $\beta=\{w \in \mathbb{C}:|w|=1+\delta\}$ and $\delta$ is a positive constant such that $1+\delta$ $<\min \left\{2,1 /\|\mu\|_{\infty}\right\}$. Then by a straightforward computation, we know that for any fixed $z \in \Delta^{*}$,

$$
\frac{d^{k} \Phi(t \mu)(z)}{d t^{k}}=\frac{k !}{2 \pi i} \int_{\beta} \frac{\Phi(w \mu)(z)}{(w-t)^{k+1}} d w,|t|<1+\delta, \quad k=1,2, \ldots
$$


Now we shall show by induction that for any fixed $z \in \Delta^{*}$,

$$
d^{k} \Phi(t \mu ; \underbrace{\mu, \ldots, \mu}_{k})(z)=\frac{d^{k} \Phi(t \mu)(z)}{d t^{k}},|t|<1+\delta, k=1,2, \ldots
$$

At first, by (5), (6) and Lemma 5 we have

$$
\begin{aligned}
\left\|\Phi(t \mu+\tau \mu)(z)-\Phi(t \mu)-\tau \cdot \frac{d \Phi(t \mu)(z)}{d t}\right\|_{h} & =\left\|\frac{\tau^{2}}{2 \pi i} \int_{\beta} \frac{\Phi(w \mu)(z)}{[w-(t+\tau)](w-t)^{2}} d w\right\|_{h} \\
& \leqslant \frac{6 \tau^{2}}{(1+\delta-|t|)^{3}} \\
& =o(\tau)
\end{aligned}
$$

when $|\tau| \rightarrow 0+$ and $|\tau|<(1+\delta-|t|) / 2$. So by the definition of Gâteaux derivatives, we know that for any fixed $z \in \Delta^{*}$,

$$
d \Phi(t \mu ; \mu)(z)=\frac{d \Phi(t \mu)(z)}{d t},|t|<1+\delta
$$

Suppose that (7) holds for $k=n$. Then by this assumption, (6) and Lemma 5 , we can also obtain

$$
\left\|d^{n} \Phi(t \mu+\tau \mu ; \underbrace{\mu, \ldots, \mu}_{n})(z)-d^{n} \Phi(t \mu ; \underbrace{\mu, \ldots, \mu}_{n})(z)-\tau \cdot \frac{d^{n+1} \Phi(t \mu)(z)}{d t^{n+1}}\right\|_{h}=o(\tau)
$$

when $|\tau| \rightarrow 0+$, that is, for any fixed $z \in \Delta^{*}$,

$$
d^{n+1} \Phi(t \mu ; \underbrace{\mu, \ldots, \mu}_{n+1})(z)=\frac{d^{n+1} \Phi(t \mu)(z)}{d t^{n+1}},|t|<1+\delta .
$$

Then (7) follows.

Especially, by (7) and (6) we have

$$
\begin{aligned}
d^{k} \Phi(0 ; \underbrace{\mu, \ldots, \mu}_{k})(z) & =\left.\frac{d^{k} \Phi(t \mu)(z)}{d t^{k}}\right|_{t=0} \\
& =\frac{k !}{2 \pi i} \int_{\beta} \frac{\Phi(w \mu)(z)}{w^{k+1}} d w, \quad k=1,2, \ldots
\end{aligned}
$$


Hence for any fixed $z \in \Delta^{*}$ and any $c \in \mathbb{C},|c|<1$,

$$
\begin{aligned}
& \left|\Phi(c \mu)(z)-\Phi(0)(z)-d \Phi(0 ; \mu)(z) \cdot c-\cdots-d^{n} \Phi(0 ; \underbrace{\mu, \ldots, \mu}_{n})(z) \cdot \frac{c^{n}}{n !}\right| \\
& \quad \leqslant \frac{1}{2 \pi} \int_{\beta}|\Phi(w \mu)(z)| \cdot\left|\frac{1}{w-c}-\frac{1}{w}-\frac{c}{w^{2}}-\cdots-\frac{c^{n}}{w^{n+1}}\right| \cdot|d w| \\
& \quad=\frac{1}{2 \pi} \int_{\beta}|\Phi(w \mu)(z)| \cdot \frac{|c|^{n+1}}{|w-c||w|^{n+1}} \cdot|d w| \\
& \quad \leqslant \frac{|c|^{n+1}}{2 \pi \delta} \int_{\beta}|\Phi(w \mu)(z)||d w| .
\end{aligned}
$$

Then by Lemma 5 , we obtain that for any positive integer $n$ and any $c \in \mathbb{C},|c|<1$,

$$
\left\|\Phi(c \mu)-\Phi(0)-d \Phi(0 ; \mu) \cdot c-\cdots-d^{n} \Phi(0 ; \underbrace{\mu, \ldots, \mu}_{n}) \cdot \frac{c^{n}}{n !}\right\|_{h} \leqslant \frac{3}{\delta}|c|^{n+1} .
$$

Theorem 3. Let $\mu \in B(\Gamma)$ be fixed and $\varphi_{n}, n=1,2, \ldots$, be defined as in (1). Suppose $a_{n}(z)=T \varphi_{n}(z), z \in \Delta^{*}, n=1,2, \ldots$ Then for any $z \in \Delta^{*}$,

$$
\begin{aligned}
d \Phi(0 ; \mu)(z) & =a_{1}^{\prime \prime \prime}(z), \\
d^{k} \Phi(0 ; \underbrace{\mu, \ldots, \mu}_{k})(z) & =\left[a_{k}^{\prime \prime \prime}(z)-P_{k}\left(a_{1}^{\prime}(z), a_{1}^{\prime \prime}(z), a_{1}^{\prime \prime \prime}(z), \ldots, a_{k-1}^{\prime}(z), a_{k-1}^{\prime \prime}(z), a_{k-1}^{\prime \prime \prime}(z)\right)\right] \cdot k !, \\
k & =2,3, \ldots,
\end{aligned}
$$

where $P_{k}\left(x_{1}, x_{2}, \ldots, x_{3 k-3}\right), k=2,3, \ldots$, are polynomials of order $k$ of $x_{1}, x_{2}, \ldots$, $x_{3 k-3}$, and independent of $\mu$.

Proof: By Lemma 1 , for any $t \in \mathbb{C},|t|<1 /\|\mu\|_{\infty}$, we have

$$
\tilde{f}_{t \mu}(z)=z+\sum_{n=1}^{\infty} t^{n} a_{n}(z), \quad \forall z \in \mathbb{C},
$$

and the series $\sum_{n=1}^{\infty} a_{n}(z)$ is absolutely and uniformly convergent in $\mathbb{C}$. Then for any $z \in \Delta^{*}$ and any $t \in \mathbb{C},|t|<1$,

$$
\begin{aligned}
\frac{d \tilde{f}_{t \mu}(z)}{d z} & =1+\sum_{n=1}^{\infty} t^{n} a_{n}^{\prime}(z) \\
\frac{d^{2} \tilde{f}_{t \mu}(z)}{d z^{2}} & =\sum_{n=1}^{\infty} t^{n} a_{n}^{\prime \prime}(z) .
\end{aligned}
$$


Thus by a straightforward computation, we have

$$
\frac{d^{2} \tilde{f}_{t \mu}(z)}{d z^{2}} / \frac{d \tilde{f}_{t \mu}(z)}{d z}=\sum_{n=1}^{\infty} t^{n} b_{n}(z),
$$

where

$$
\begin{aligned}
b_{1}(z) & =a_{1}^{\prime \prime}(z) \\
b_{2}(z) & =a_{2}^{\prime \prime}(z)-a_{1}^{\prime \prime}(z) a_{1}^{\prime}(z) \\
& \ldots \ldots \\
b_{n}(z) & =a_{n}^{\prime \prime}(z)-\widetilde{P}_{n}\left(a_{1}^{\prime}(z), a_{1}^{\prime \prime}(z), \ldots, a_{n-1}^{\prime}(z), a_{n-1}^{\prime \prime}(z)\right) \\
& \ldots \cdots
\end{aligned}
$$

and $\widetilde{P}_{n}\left(x_{1}, x_{2}, \ldots, x_{2 n-2}\right), n=2,3, \ldots$, are polynomials of order $n$ of $x_{1}, x_{2}, \ldots$, $x_{2 n-2}$, which are independent of $\mu$. Hence for any $z \in \Delta^{*}$ and any $t \in \mathbb{C},|t|<1$,

$$
\Psi(t \mu)(z)=S_{\left.\widetilde{f}_{\mu}\right|_{\Delta^{*}}}=\sum_{n=1}^{\infty} t^{n} \widetilde{b}_{n}(z)
$$

where

$$
\begin{aligned}
\tilde{b}_{1}(z) & =a_{1}^{\prime \prime \prime}(z), \\
\tilde{b}_{2}(z) & =a_{2}^{\prime \prime \prime}(z)-a_{1}^{\prime \prime \prime}(z) a_{1}^{\prime}(z)-\frac{3}{2}\left(a_{1}^{\prime \prime}(z)\right)^{2}, \\
\ldots & \cdots, \\
\tilde{b}_{n}(z) & =a_{n}^{\prime \prime \prime}(z)-P_{n}\left(a_{1}^{\prime}(z), a_{1}^{\prime \prime}(z), a_{1}^{\prime \prime \prime}(z), \ldots, a_{n-1}^{\prime}(z), a_{n-1}^{\prime \prime}(z), a_{n-1}^{\prime \prime \prime}(z)\right),
\end{aligned}
$$

and $P_{n}\left(x_{1}, x_{2}, \ldots, x_{3 n-3}\right), n=2,3, \ldots$, are polynomials of order $n$ of $x_{1}, x_{2}, \ldots$, $x_{3 n-3}$, which are independent of $\mu$. Thus by (8) in the proof of Theorem 2 and Lemma 7 , we can obtain that for any fixed $z \in \Delta^{*}$,

$$
\begin{aligned}
d^{k} \Phi(0 ; \underbrace{\mu, \ldots, \mu}_{k})(z) & =\left.\frac{d^{k} \Phi(t \mu)(z)}{d t^{k}}\right|_{t=0} \\
& =\left.\frac{d^{k} \Psi(t \mu)(z)}{d t^{k}}\right|_{t=0} \\
& =\widetilde{b}_{k}(z) \cdot k !, \quad k=1,2, \ldots
\end{aligned}
$$

This completes the proof of Theorem 3 . 


\section{Proof of Theorem 1}

Let

$$
\varphi_{1}=\mu_{1}, \quad \widetilde{\varphi}_{1}=\mu_{2}, \quad \varphi_{n}=\mu_{1} H\left(\varphi_{n-1}\right), \quad \tilde{\varphi}_{n}=\mu_{2} H\left(\tilde{\varphi}_{n-1}\right), \quad n=2,3, \ldots,
$$

and

$$
a_{n}(z)=T \varphi_{n}(z), \quad \tilde{a}_{n}=T \tilde{\varphi}_{n}(z), \quad z \in \Delta^{*}, n=1,2, \ldots
$$

Then by Theorem 2, for any positive integer $n$ and any $c \in \mathbb{C},|c|<1$, we have the following inequalities:

(9)

(10)

$$
\left\|\Phi\left(c \mu_{1}\right)-\Phi(0)-d \Phi\left(0 ; \mu_{1}\right) \cdot c-\cdots-d^{n} \Phi(0 ; \underbrace{\mu_{1}, \ldots, \mu_{1}}_{n}) \cdot \frac{c^{n}}{n !}\right\|_{h} \leqslant \frac{3}{\delta}|c|^{n+1},
$$

$$
\left\|\Phi\left(c \mu_{2}\right)-\Phi(0)-d \Phi\left(0 ; \mu_{2}\right) \cdot c-\cdots-d^{n} \Phi(0 ; \underbrace{\mu_{2}, \ldots, \mu_{2}}_{n}) \cdot \frac{c^{n}}{n !}\right\|_{h} \leqslant \frac{3}{\delta}|c|^{n+1}
$$

where $\delta$ is a positive constant only depending on $\left\|\mu_{1}\right\|_{\infty}=\left\|\mu_{2}\right\|_{\infty}$.

Suppose that two geodesic segments $\left[t \mu_{1}\right](0 \leqslant t \leqslant 1)$ and $\left[t \mu_{2}\right](0 \leqslant t \leqslant 1)$ are the same. They are both geodesic segments joining $[0]$ and $[\mu]$, and $d_{T}\left(\left[t \mu_{1}\right],[0]\right)$ $=d_{T}\left(\left[t \mu_{2}\right],[0]\right)(0 \leqslant t \leqslant 1)$ because of extremality of $\mu_{1}$ and $\mu_{2}([10])$. Then

$$
\left[t \mu_{1}\right]=\left[t \mu_{2}\right], \quad 0 \leqslant t \leqslant 1
$$

So by Lemma 4, we know that

$$
\Phi\left(t \mu_{1}\right)=\Phi\left(t \mu_{2}\right) \quad(0 \leqslant t \leqslant 1) \text { in } H Q D\left(\Gamma^{*}\right)
$$

Setting $n=1$ in (9) and (10), by the triangle inequality of norms we have

$$
\begin{aligned}
\left\|d \Phi\left(0 ; \mu_{1}\right)-d \Phi\left(0 ; \mu_{2}\right)\right\|_{h} \cdot|c| & \leqslant\left\|\Phi\left(c \mu_{1}\right)-\Phi\left(c \mu_{2}\right)\right\|_{h}+2 \times \frac{3}{\delta}|c|^{2} \\
& =\frac{6}{\delta}|c|^{2}, \quad 0<c<1 .
\end{aligned}
$$

Let $c \rightarrow 0$. Hence

$$
\left\|d \Phi\left(0 ; \mu_{1}\right)-d \Phi\left(0 ; \mu_{2}\right)\right\|_{h}=0
$$

that is,

$$
d \Phi\left(0 ; \mu_{1}\right)(z)=d \Phi\left(0 ; \mu_{2}\right)(z), \quad \forall z \in \Delta^{*}
$$


By induction, from (9) and (10) we can similarly deduce that

$$
d^{n} \Phi(0 ; \underbrace{\mu_{1}, \ldots, \mu_{1}}_{n})(z)=d^{n} \Phi(0 ; \underbrace{\mu_{2}, \ldots, \mu_{2}}_{n})(z), \quad \forall z \in \Delta^{*}, n=2,3, \ldots
$$

Thus by Theorem 3, we know that for any $z \in \Delta^{*}$,

$$
a_{1}^{\prime \prime \prime}(z)=\tilde{a}_{1}^{\prime \prime \prime}(z),
$$

and

$$
\begin{aligned}
& a_{n}^{\prime \prime \prime}(z)-P_{n}\left(a_{1}^{\prime}(z), a_{1}^{\prime \prime}(z), a_{1}^{\prime \prime \prime}(z), \ldots, a_{n-1}^{\prime}(z), a_{n-1}^{\prime \prime}(z), a_{n-1}^{\prime \prime \prime}(z)\right) \\
= & \widetilde{a}_{n}^{\prime \prime \prime}(z)-P_{n}\left(\widetilde{a}_{1}^{\prime}(z), \widetilde{a}_{1}^{\prime \prime}(z), \tilde{a}_{1}^{\prime \prime \prime}(z), \ldots, \tilde{a}_{n-1}^{\prime}(z), \widetilde{a}_{n-1}^{\prime \prime}(z), \widetilde{a}_{n-1}^{\prime \prime \prime}(z)\right), \quad n=2,3, \ldots,
\end{aligned}
$$

where $P_{n}\left(x_{1}, x_{2}, \ldots, x_{3 n-3}\right), n=2,3, \ldots$, are polynomials of order $n$ of $x_{1}, x_{2}, \ldots$, $x_{3 n-3}$, and independent of $\mu_{1}$ and $\mu_{2}$.

Next we shall prove by induction that for any integer $n$,

$$
a_{n}(z)=\tilde{a}_{n}(z), \quad \forall z \in \Delta^{*} .
$$

At first, by (11) we know that (13) holds for $n=1$.

Suppose that (13) holds for $n \leqslant k$. Then by the equivalence of (A2) and (A3) and the equivalence of (B2) and (B3) in Lemma 6, from (12) we can obtain that (13) also holds for $n=k+1$. Hence by induction, (13) holds for any integer $n$, that is,

$$
T\left(\varphi_{n}-\tilde{\varphi}_{n}\right)(z)=0, \quad \forall z \in \Delta^{*}, n=1,2, \ldots
$$

Furthermore, by the equivalence of (A1) and (A2) and the equivalence of (B1) and (B2) in Lemma 6 and noting that $\varphi_{1}-\widetilde{\varphi}_{1} \in \operatorname{Belt}(\Gamma)$ and $\varphi_{n}-\widetilde{\varphi}_{n} \in \operatorname{Belt}^{*}(1), n$ $=2,3, \ldots$, from $(14)$ we have

$$
\varphi_{1}-\tilde{\varphi}_{1} \in N(\Gamma)
$$

and

$$
\varphi_{n}-\tilde{\varphi}_{n} \in N^{*}(1), \quad n=2,3, \ldots .
$$

Conversely, suppose that $\varphi_{1}-\widetilde{\varphi}_{1} \in N(\Gamma)$ and $\varphi_{n}-\tilde{\varphi}_{n} \in N^{*}(1), n=2,3, \ldots$. Then by the equivalence of (A1) and (A2) and the equivalence of (B1) and (B2) in Lemma 6 and noting that $\varphi_{1}-\widetilde{\varphi}_{1} \in \operatorname{Belt}(\Gamma)$ and $\varphi_{n}-\widetilde{\varphi}_{n} \in \operatorname{Belt}^{*}(1), n=2,3 \ldots$. we know that

$$
T\left(\varphi_{n}-\widetilde{\varphi}_{n}\right)(z)=0, \quad \forall z \in \Delta^{*}, n=1,2, \ldots,
$$

that is,

$$
a_{n}(z)=\tilde{a}_{n}(z), \quad \forall z \in \Delta^{*}, n=1,2, \ldots
$$


Thus by Theorem 3, the equivalence of (A2) and (A3) and the equivalence of (B2) and (B3) in Lemma 6. we deduce that

$$
d^{n} \Phi(0 ; \underbrace{\mu_{1}, \ldots, \mu_{1}}_{n})(z)=d^{n} \Phi(0 ; \underbrace{\mu_{2}, \ldots, \mu_{2}}_{n})(z), \quad \forall z \in \Delta^{*}, n=1,2, \ldots .
$$

Hence by means of (9), (10) and the triangle inequality of norms, we obtain that

$$
\begin{aligned}
\left\|\Phi\left(c \mu_{1}\right)-\Phi\left(c \mu_{2}\right)\right\|_{h} & \leqslant 2 \times \frac{3}{\delta}|c|^{n+1} \\
& =\frac{6}{\delta}|c|^{n+1}, \quad n=1,2, \ldots .
\end{aligned}
$$

Let $n \rightarrow+\infty$. Then we have

$$
\left\|\Phi\left(c \mu_{1}\right)-\Phi\left(c \mu_{2}\right)\right\|_{h}=0, \quad \forall c, 0<c<1
$$

that is,

$$
\Phi\left(c \mu_{1}\right)=\Phi\left(c \mu_{2}\right), \quad \forall c, 0<c<1 .
$$

So by Lemma 4 , we know that for any $c, 0 \leqslant c \leqslant 1$,

$$
\left[c \mu_{1}\right]=\left[c \mu_{2}\right] \text { in } T(\Gamma) \text {. }
$$

Hence, two geodesic segments $\left[t \mu_{1}\right](0 \leqslant t \leqslant 1)$ and $\left[t \mu_{2}\right](0 \leqslant t \leqslant 1)$ are the same.

\section{REFERENCES}

[1] H. Busemann, The geometry of geodesics (Academic Press, New York, 1955).

[2] D. Gaier, Lectures on complex approximation (Birkhäuser, Boston, 1987).

[3] F. P. Gardiner, 'On completing triangles in infinite dimensional Teichmüller spaces', Complex Variables Theory Appl. 10 (1988), 237-247.

[4] Y. Imayoshi and M. Taniguchi, An introduction to Teichmüller spaces (Spring-Verlag, Tokyo, 1992).

i5! S. Kravetz, On the geometry of Teichmüller spaces and the structure of their modular groups', Ann. Acad. Sci. Fenn. Ser. A I Math. 278 (1959), 1-35.

[6] O. Lehto, Univalent functions and Teichmüller spaces, Graduate Texts in Mathematics 109 (Springer-Verlag, New York, 1987).

(7] Z. Li, Quasiconformal mappings and their applications in the theory of Riemann surfaces, (in Chinese) (Scientific Press, Beijing, 1988).

[8] Z. Li, 'Non-uniqueness of geodesics in infinite dimensional Teichmiiller spaces', Complex Variables Theory Appl. 16 (1991), 261-272.

[9] Z. Li, 'Non-uniqueness of geodesics in infinite dimensional Teichmüller spaces (II)', Ann. Acad. Sci. Fenn. Ser. A I Math. 18 (1993), 355-367. 
[10] B. O'Byrne, On Finsler geometry and applications to Teichmüller spaces, Ann. of Math. Studies 66 (Princeton University Press, Princeton, NJ, 1971), pp. 317-328.

[11] Y. Shen, 'Non-uniqueness of geodesics in the universal Teichmüller spaces', Adv. in Math. (China) 24 (1995), 237-243.

[12] K. Strebel, 'Extremal quasiconformal mappings', Resultate Math. 10 (1986), 168-210.

[13] H. Tanigawa, 'Holomorphic families of geodesic discs in infinite dimensional Teichmüller spaces', Nagoya Math. J. 127 (1992), 117-128.

Department of Mathematical Education

Normal College

Shenzhen University

Shenzhen, Guangdong 518060

Peoples Republic of China
Institute of Mathematics

Chinese Academy of Sciences

Beijing 100080

Peoples Republic of China

e-mail: hguo@szu.edu.cn

szuhguo@hotmail.com 